\title{
Quantum Domains for Macroscopic Transport Effects in Nanostructures with Control Topology: Optics and e- Conductivity
}

\author{
A. Antipov, S. Arakelian" , A. Kucherik, S. Kutrovskaya, D. Nogtev, A. Osipov, T. Vartanyan, \\ and S. Zimin ${ }^{2}$ \\ Vladimir State University named after A.G. and N.G. Stoletovs, Vladimir, 600000, Russia \\ ${ }^{1}$ St. Petersburg National Research University of Information Technologies, Mechanics and Optics, St. Petersburg, \\ 197101 Russia \\ ${ }^{2}$ P.G. Demidov Yaroslavl State University, Yaroslavl, 150000, Russia
}

\begin{abstract}
The nanostructures with different morphology have been obtained by us by methods of both direct laser modification (from $\mathrm{cw}$ to fs laser radiation) of the target surface/thin films and laser evaporation of the target substance in liquid to produce the colloid systems, and then - to deposite substance on substrate from colloid, and also - by a single drop deposition technique. The analysis of induced nanostructures has been carried out by absorption spectroscopy, scanning electron microscopy and transmission electron microscopy. The island conductivity is dominant for the nanocluster semiconductor systems induced by laser ablation technique, and electroresistance can dramatically decrease due to spontaneous selected multichannel/parallel electron transportation trajectories. A tunneling quantum coherent effect takes place for electron conductivity for the case.
\end{abstract}

Keywords: quantum dots, nanocluster structure, jump electroconductivity

1. The paper represents the results of laser synthesis of semiconductor nanoparticles of PbTe by methods of both laser modification of thin films and laser evaporation of substance in liquid to produce the colloidal systems. Under a cw-laser radiation the nanoparticles become quantum dots. By drop deposition technique it has been obtained the structures with various morphology which depends on the substrate temperature. The optical and electro-physical properties of the structures can be controlled, and are very important to construct the devices of optoelectronics and photonics on new physical principles.

2. The cw-laser ablation experiments $\left(\lambda=1.06 \mu \mathrm{m}\right.$, laser intensity - up to $\left.10^{6} \mathrm{~W} / \mathrm{cm}^{2}\right)$ with colloidal systems to induce the surface semiconductor nanostructure on substrate have been carry out to study electrical transport properties under quantum tunneling effect.

Comparison of sizes $R_{0}$ of the particles being obtained with the value of exciton Bohr radius $a_{B}$ shows that the strict condition $R_{0} \leq a_{B}=50-100 \mathrm{~nm}$ is true. The above comparison enables to say that the conditions of dimensional quantization are satisfied for PbTe nanoparticles. On the other hand,

${ }^{a}$ Corresponding author: arak@vlsu.ru

This is an Open Access article distributed under the terms of the Creative Commons Attribution License 4.0, which permits unrestricted use, distribution, and reproduction in any medium, provided the original work is properly cited. 
when the nanoparticles drop on the solid surface they are accumulated in clusters of bigger size (see Fig. 1a). So, we can study the macroscopic quantum phenomena development in dependence on $R_{0}$.

3. The main conclusion of our study is that the island conductivity is dominant for the case. An electroresistance can dramatically decrease due to spontaneous selected multichannel/parallel electron transportation trajectories (Fig. 2). Two conditions are the vital items for that: the cluster size $a<l$, where $l$ is the inelastic length, and distance $a$ between two neighboring clusters less the de Broglie wavelength $\lambda_{\mathrm{dB}}$. So, the tunneling quantum effect takes place.

For such nanostructures we demonstrated the superconductivity tendency (Fig. 1b) to increase the electrical conductivity (in several times for our case) at room temperature in comparison with homogenous sample. The fact can be explained in analogy with correlated particles/coupling pairs from two sides of the border for double electric layer/two barriers due to quantum hole being under a coherent tunnel effect.

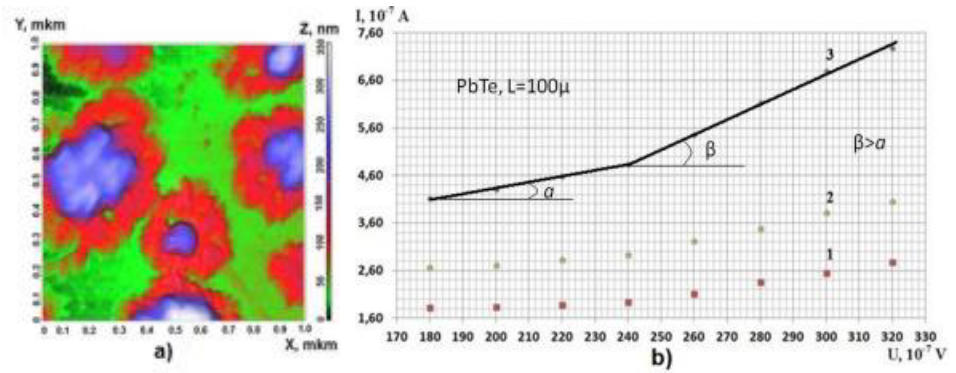

Figure. 1. (a) AFM-image of deposited PbTe-clusters and (b) the Volt-Amper experimental data view in the frames of the tunneling mechanism conductivity for $\mathrm{PbTe}$ deposited layers under average cluster size $l$ and average distance between clusters (a), correspondingly: (1) $120 \mathrm{~nm}$ and $20 \mathrm{~nm}$; (2) $210 \mathrm{~nm}$ and $8 \mathrm{~nm}$; (3) $120 \mathrm{~nm}$ and $8 \mathrm{~nm}$. The electroresistance dependence $\mathrm{R}=\mathrm{U} / \mathrm{I}=\operatorname{ctg}(\alpha, \beta)$ (less $\mathrm{R}$ more $a \beta$ ) means more tunneling impact in the case (3).

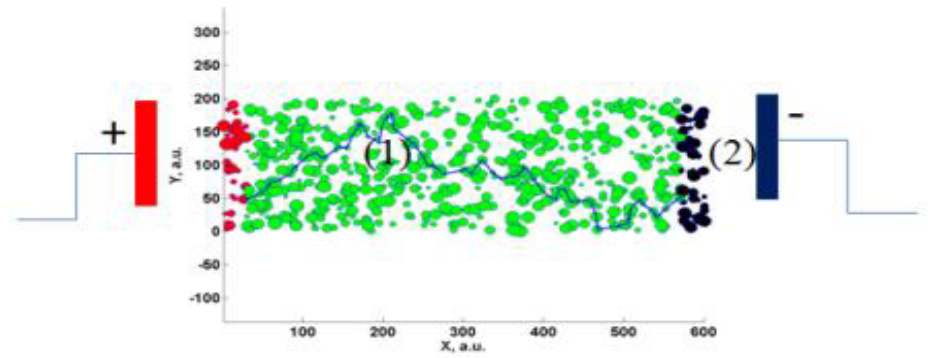

Figure. 2. Spontaneous conductivity channels for semiconductor grain layer: modeling for both (1) a single electron transportation trajectory and (2) a summarize electro conductivity for different trajectories over crosssection. 